\title{
PARALLEL LOGICS USING MULTIMODE EXCITATION OF A SINGLE MEMS RESONATOR
}

\author{
Nizar Jaber ${ }^{1,2}$, Saad Ilyas ${ }^{1}$, and Mohammad I. Younis ${ }^{1, *}$ \\ ${ }^{1}$ Physical Sciences and Engineering Division, King Abdullah University of Science and Technology, \\ Thuwal, Saudi Arabia and \\ ${ }^{2}$ School of Mechanical Engineering, Purdue University, West Lafayette, IN, USA
}

\begin{abstract}
Performing Boolean operations using conventional CMOS-based computers requires the wiring of multiple transistors. Hence, the processors of these computers are composed of millions of transistors, which increase the device size and power consumption. Here, we present a single MEMS resonator that can execute in parallel multiple logic gates. The concept is based on simultaneously encoding the binary information at different modes of vibration of a microplate. The proposed novel method allows the device not only to perform as a fully integrated logic gate but also as a parallel logic processor for which the same device can perform multiple logic gates simultaneously. The proposed method decreases the device footprint and reduces the power consumption required to perform multiple Boolean functions.
\end{abstract}

\section{KEYWORDS}

Mechanical computations, parallel logic, multimode resonator, microplate.

\section{INTRODUCTION}

In traditional transistor-based computers, connecting different transistors is essential to execute logic operations, which increases the device size and energy consumption. Also, the transistor technology is reaching its physical limit. Hence, different alternatives are being sought to execute logic operations [1-6]. Motivated by their low power consumptions and robustness to electromagnetic shock, recently, micromechanical structures have been employed to demonstrate universal logic gates at which the computation can be carried out in two different forms, static and dynamic mode [1]. In the static mode, relays or MEMS switches are employed to execute the logic functions in which the logic states are realized by selectively actuating the switches. This mode has the advantages of zero leakage current and the ability to easily cascade multiple elements to execute complex logic operations. However, their high contact resistance, low speed, and stiction issues limit their potential in demonstrating practical applications $[7,8]$. Therefore, a new research direction has emerged focusing on the noncontact mode by employing the dynamic vibration of MEMS and NEMS resonators. In the dynamic mode, the logic states are encoded by activating and deactivating the resonance frequency using different modulation and actuation techniques, such as DC tuning [9], electrothermal tuning $[10,11]$, parametric resonance [12], and frequency mixing [13]. Utilizing different modes of vibration to execute logic operations improves the operation speed of the logic unit and increases the lifetime of the proposed device. The enhanced functionality of the resonator-based computing has been demonstrated by showing a reconfigurable MEMS resonator that can perform several universal logic gates using a single MEMS resonator [10]. However, the difference between the input and output frequencies complicates the possibility of cascading multiple devices to perform complex logic functions. Recently, the potential for cascading multiple logic elements has been demonstrated by operating different resonators at the same frequency and using the AC signal to represent the logic inputs [14]. Multiple logic gates are demonstrated by selectively activating and deactivating different modes of vibrations of a piezoelectric circular membrane [15]. Also, by utilizing parametric resonance, the possibility of realizing parallel logic is demonstrated [3]. However, the need for extremely controlled test conditions and the small frequency difference between the parallel logic outputs $(\sim$ $1 \mathrm{~Hz}$ ) hinder their potential in practical application.

Recently, exploiting information from the different modes of vibration of a single MEMS resonator has gained significant attention in wide range of applications, such as the simultaneous measurements of temperature and humidity using two modes [16], extracting the mechanical properties and mass of adsorbed particles [17], determining the position and mass of adsorbed analytes [18, 19], and demonstrating a smart sensor that can autonomously activate a switch upon exceeding a threshold value [20].

Here, we demonstrate an electrostatically actuated microplate resonator, simultaneously actuated near the first four modes of vibration, which can perform various logic gates in parallel (simultaneously). Each gate is operated at a single mode. Hence, by using the appropriate electrical interconnect, multiple devices can be cascaded to demonstrate complex logic operations.

\section{EXPERIMENTAL SETUP}

As shown in Fig. 1a, the microplate, which forms the upper electrode, is fabricated from a $4.3 \mu \mathrm{m}$ polyimide layer coated with metals from top and bottom. The upper electrode is perforated to reduce the effect of squeeze film damping and decrease the time required to fully release the device. The microplate length $(l)$ is $400 \mu \mathrm{m}$ and of width $(w) 150 \mu \mathrm{m}$. The perforation diameter $(d)$ is $60 \mu \mathrm{m}$ with spacing $(S)$ of $50 \mu \mathrm{m}$. The lower electrode is divided into six different electrodes that can be separately accessed. By powering specific lower electrode configuration, we can selectively activate and deactivate particular modes of vibration. The experimental setup is shown in Fig. 1b. The microbeam is electrostatically actuated by a data acquisition card that provides a multi- 
frequency signal. A laser Doppler vibrometer is utilized to monitor the beam response.

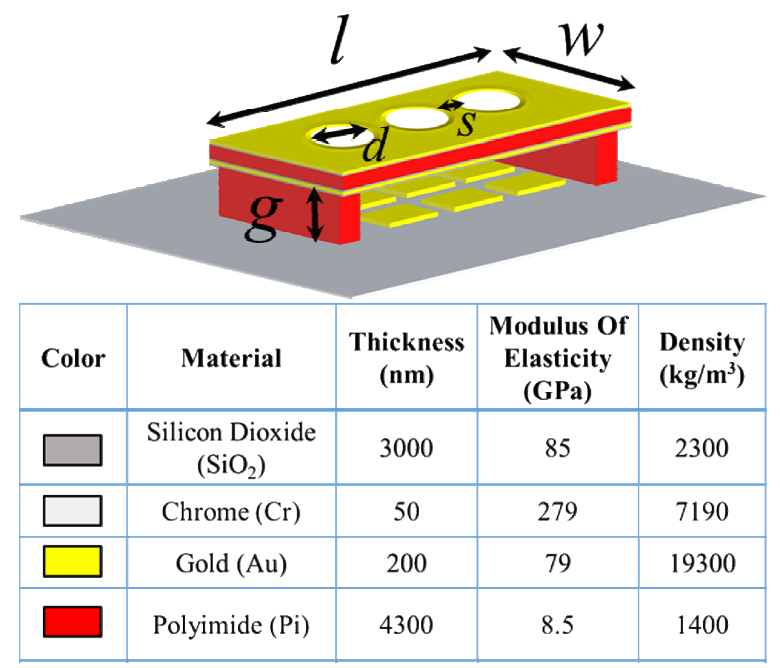

(a)

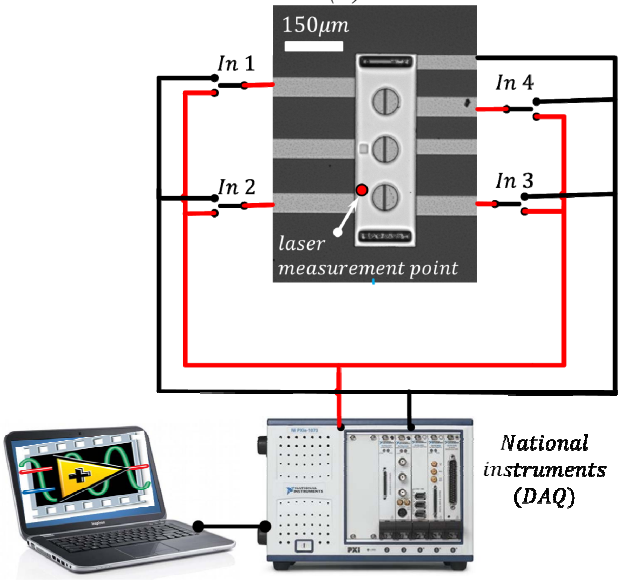

(b)

Figure 1: (a) Schematic of the microplate with lower partial electrodes. (b) The experimental setup showing the microplate, data acquisition card, and the four input switches used to provide the logic inputs.

\section{RESULTS AND DISCUSSION}

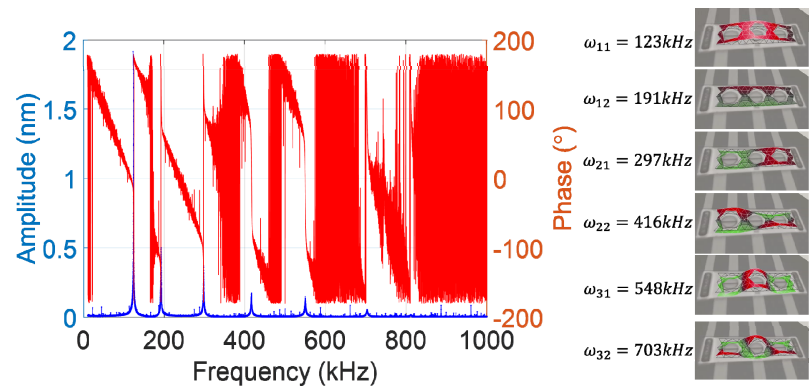

Figure 2: Frequency response of the microplate to a white noise excitation at $V_{D C}=10 \mathrm{~V}, V_{A C}=15 \mathrm{~V}$, and at a chamber pressure $P=4$ mTorr. Insets: the corresponding mode shapes acquired by recording the response at different points along the microplate surface using a laser Doppler vibrometer.

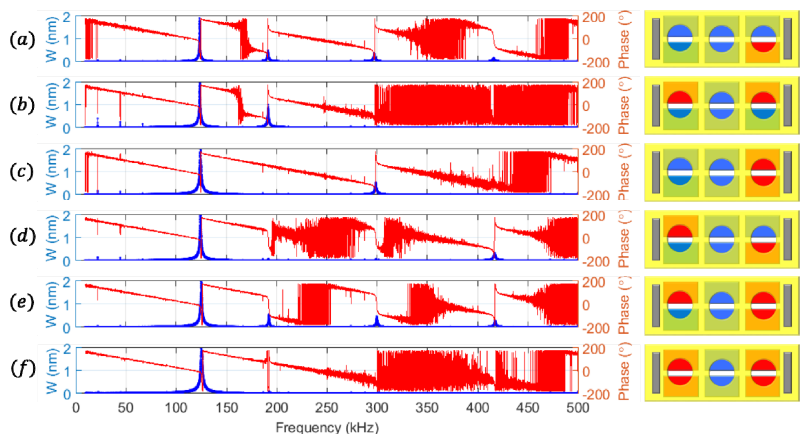

Figure 3: Modes activation and deactivation by powering different lower electrode configuration to a white noise excitation at $V_{D C}=10 \mathrm{~V}, V_{A C}=15 \mathrm{~V}$, and at a chamber pressure $P=4$ mTorr. (a) One electrode. (b) Two-side electrodes. (c) Two-anchor electrodes. (d) Two-diagonal electrodes. (e) Three electrodes. (f) Four electrodes.

The resonance frequencies and their corresponding mode shapes are revealed by using one electrode to actuate the microplate with white noise signal while recording the vibrations at different points along the microplate surface, Fig. 2. As shown in Fig.2, the first six modes can be excited using any of the corner electrodes. In this study, we utilize the corner electrodes to selectively activate the first four modes. To demonstrate the concept, we powered different lower electrode configuration with the white noise signal and recorded the microplate response, Fig.3. As shown in Fig. 3a and 3e, the four modes can be excited using one electrode or three electrodes. By powering the side electrodes, Fig. 3b, the first and second modes are activated. Connecting the twoanchor electrodes activates the first and third mode, Fig. $3 c$. Figure $3 d$ shows that the first and fourth mode can be activated by powering the diagonal electrodes. As depicted in Fig. 3e, powering the four electrodes activates the first mode only. Hence, by powering different lower electrodes, which represent the logic inputs, certain modes can be selectively activated/deactivated, which represents the logic output.

Figure 4 shows the results of sweeping the frequency response near the first four modes of vibration. This test is important in determining the appropriate voltage required to activate the different modes with a high signal-to-noise ratio. To demonstrate the parallel logic operation, we utilize the four corner electrodes to actuate the microplate with a multi-frequency electrical signal that has frequencies matching the first four modes. Then, to realize the different possible logic gates, we monitor the response to different input combinations by connecting and disconnecting the input switches shown in Fig. 1b. The results are demonstrated in Fig. 4 for the 16 possible input combinations. For example, by considering Fig. 4a, we demonstrate the possibility of realizing OR gates at $\omega_{11}$ and $\omega_{12}$, and also XOR gates at $\omega_{21}$ and $\omega_{22}$. The logic low state ' 0 ' is defined for vibration amplitudes less than 10 $\mathrm{nm}$, and the logic high state ' 1 ' is defined for any response above $10 \mathrm{~nm}$. The reported results show the possibility of realizing XNOR and NOT gates in parallel as shown in Fig. 4b and Fig. 4c. Figure 4d shows the potential of performing NAND and two XOR gates simultaneously. 


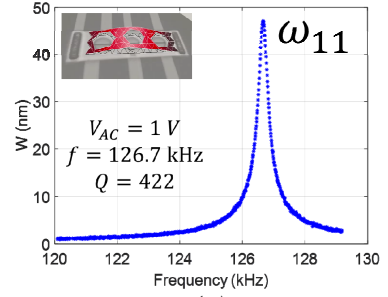

(a)

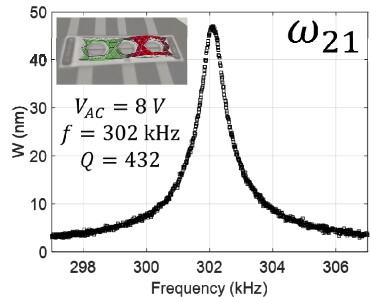

(c)

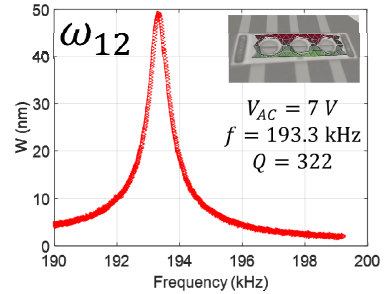

(b)

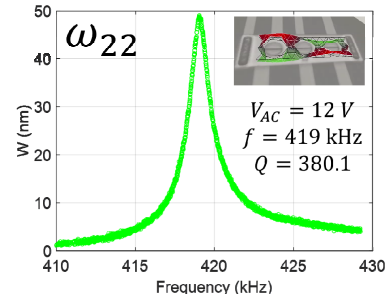

(d)

Figure 4: frequency response of the microplate at $V_{D C}=5 \mathrm{~V}$ and a chamber pressure $P=4$ mTorr near the different modes of vibration. (a) The first mode $\omega_{11}$. (b) The second mode $\omega_{12}$. (c) The third mode $\omega_{21}$. (d) The fourth mode $\omega_{22}$.

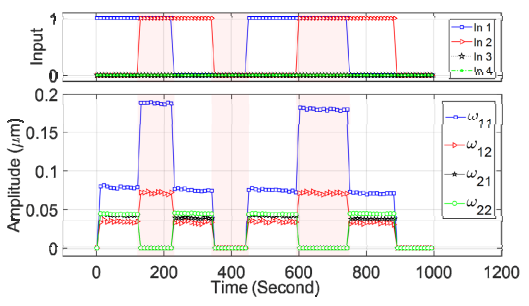

(a)

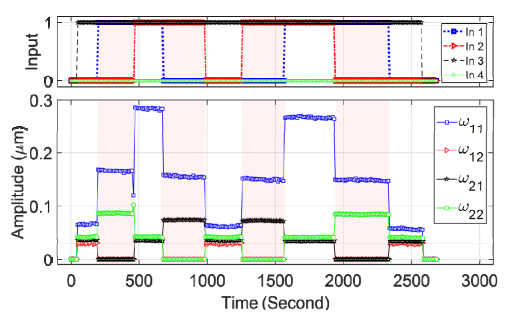

(b)

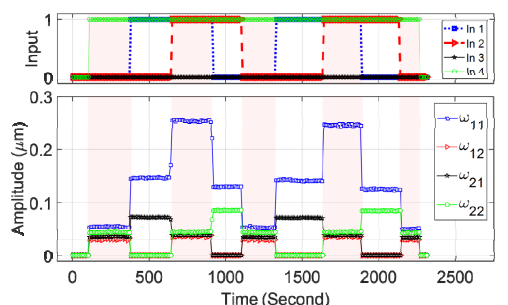

(c)

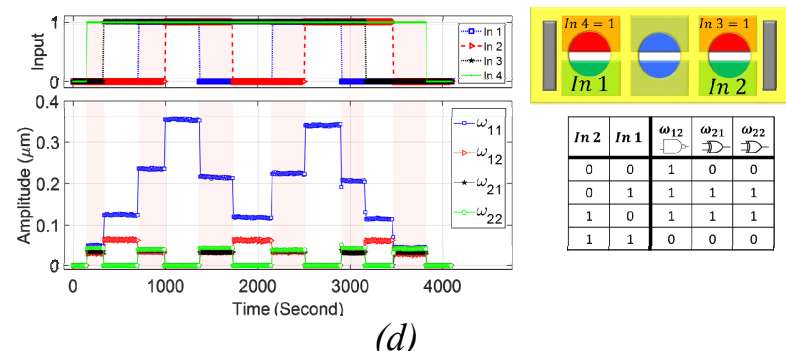

Figure 4: The response of the microplate to a multi- frequency excitation near the first four modes of vibration and different logical input configurations. (a) OR gates are demonstrated at $\omega_{11}$ and $\omega_{12}$, and $X O R$ gates are demonstrated at $\omega_{12}$. (b) XNOR gate is demonstrated at $\omega_{12}$ in parallel with a NOT gate at $\omega_{21}$. (c) XNOR gate is demonstrated at $\omega_{12}$ in parallel with a NOT gate at $\omega_{22}$. (d) NAND gate is shown at $\omega_{12}$ while XOR gates are shown at $\omega_{21}$ and $\omega_{22}$.

\section{CONCLUSIONS}

We presented a single device that can simultaneously execute fundamental logic operations. The concept is based on selectively activating and deactivating different mode vibration of a microplate, which represents the logic output, by powering different lower electrodes configurations, which represent the logic inputs. The results show the possibility of simultaneously realizing OR and XOR, XNOR and Inverter, and NAND and XOR gates. The proposed method increases the integration density and reduces the power consumption required to perform multiple Boolean functions. Further research in this device can be directed to develop the electrical readout circuit and cascade multiple devices to execute complex logic operations.

\section{ACKNOWLEDGMENTS}

This publication is based upon work supported by the King Abdullah University of Science and Technology (KAUST) under Award No. OSR-2016-CRG5-3001.

\section{REFERENCES}

[1] M. L. Roukes, "Mechanical compution, redux? [Nanoelectromechanical systems]," in IEDM Technical Digest. IEEE International Electron Devices Meeting, 2004., 2004, pp. 539-542.

[2] S. C. Masmanidis, R. B. Karabalin, I. De Vlaminck, G. Borghs, M. R. Freeman, and M. L. Roukes, "Multifunctional Nanomechanical Systems via Tunably Coupled Piezoelectric Actuation," Science, vol. 317, no. 5839, p. 780, 2007.

[3] D. N. Guerra, A. R. Bulsara, W. L. Ditto, S. Sinha, K. Murali, and P. Mohanty, "A NoiseAssisted Reprogrammable Nanomechanical Logic Gate," Nano Letters, vol. 10, no. 4, pp. 1168-1171, 2010/04/14 2010.

[4] R. H. Blick, H. Qin, H.-S. Kim, and R. Marsland, "A nanomechanical computer-exploring new avenues of computing," New Journal of Physics, vol. 9, no. 7, pp. 241-241, 2007/07/24 2007.

[5] I. Mahboob, M. Mounaix, K. Nishiguchi, A. Fujiwara, and H. Yamaguchi, "A multimode electromechanical parametric resonator array," Scientific Reports, Article vol. 4, p. 4448, 03/24/online 2014.

[6] S. Houri, G. Billiot, M. Belleville, A. Valentian, and H. Fanet, "Limits of CMOS Technology and Interest of NEMS Relays for Adiabatic Logic Applications," IEEE Transactions on Circuits 
and Systems I: Regular Papers, vol. 62, no. 6, pp. 1546-1554, 2015.

[7] C.-Y. Tsai and T.-L. Chen, "Design, fabrication and calibration of a novel MEMS logic gate,"

Journal of Micromechanics and

Microengineering, vol. 20, no. 9, p. 095021, 2010/08/17 2010.

[8] S. Ilyas, A. Arevalo, E. Bayes, I. G. Foulds, and M. I. Younis, "Torsion based universal MEMS logic device," Sensors and Actuators A: Physical, vol. 236, pp. 150-158, 2015/12/01/ 2015.

[9] S. Ahmed, S. Ilyas, X. Zou, N. Jaber, M. I. Younis, and H. Fariborzi, "A Compact Adder and Reprogrammable Logic Gate Using Microelectromechanical Resonators with Partial Electrodes," IEEE Transactions on Circuits and Systems II: Express Briefs, pp. 1-1, 2019.

[10] M. A. A. Hafiz, L. Kosuru, and M. I. Younis, "Microelectromechanical reprogrammable logic device," Nature Communications, Article vol. 7 , p. 11137, 03/29/online 2016.

[11] N. Alcheikh, S. A. Tella, and M. I. Younis, "An investigation into the mechanical behavior of multi- input and multi-output MEMS resonators," Sensors and Actuators A: Physical, vol. 280, pp. 309-318, 2018/09/01/ 2018.

[12] I. Mahboob, E. Flurin, K. Nishiguchi, A. Fujiwara, and H. Yamaguchi, "Interconnect-free parallel logic circuits in a single mechanical resonator," Nature communications, vol. 2, p. 198, 2011.

[13] S. Ilyas, N. Jaber, and M. I. Younis, "MEMS Logic Using Mixed-Frequency Excitation," Journal of Microelectromechanical Systems, vol. 26, no. 5, pp. 1140-1146, 2017.

[14] S. Ilyas, S. Ahmed, M. A. A. Hafiz, H. Fariborzi, and M. I. Younis, "Cascadable

microelectromechanical resonator logic gate," Journal of Micromechanics and Microengineering, vol. 29, no. 1, p. 015007 , 2018/11/302018.

[15] D. Hatanaka, I. Mahboob, H. Okamoto, K. Onomitsu, and H. Yamaguchi, "An electromechanical membrane resonator," Applied Physics Letters, vol. 101, no. 6, p. 063102, 2012.

[16] N. Jaber, S. Ilyas, O. Shekhah, M. Eddaoudi, and M. I. Younis, "Multimode MEMS Resonator for Simultaneous Sensing of Vapor Concentration and Temperature," IEEE Sensors Journal, vol. 18, no. 24, pp. 10145-10153, 2018.

[17] E. Gil-Santos et al., "Nanomechanical mass sensing and stiffness spectrometry based on twodimensional vibrations of resonant nanowires," Nature nanotechnology, vol. 5, no. 9, pp. 641645, 2010.

[18] S. Olcum, N. Cermak, S. C. Wasserman, and S. R. Manalis, "High-speed multiple-mode masssensing resolves dynamic nanoscale mass distributions," Nature communications, vol. 6, 2015.
[19] A. Bouchaala, A. Nayfeh, N. Jaber, and M. Younis, "Mass and position determination in MEMS mass sensors: a theoretical and an experimental investigation," Journal of Micromechanics and Microengineering, vol. 26, no. 10, p. 105009, 2016.

[20] N. Jaber, S. Ilyas, O. Shekhah, M. Eddaoudi, and M. I. Younis, "Multimode excitation of a metal organics frameworks coated microbeam for smart gas sensing and actuation," Sensors and Actuators A: Physical, vol. 283, pp. 254-262, 2018/11/01/ 2018.

\section{CONTACT}

*Mohammad Younis (mohammad.younis@kaust.edu.sa) B4 3035, KAUST, 23955-9600, Thuwal, Saudi Arabia. 